\title{
Ciudadanía social y violencia en las ciudades centroamericanas $^{1}$
}

\author{
Mario Lungo y RoXana Martel*
}

1. Introducción: acerca de la relación entre ciudadanía social y violencia en los ámbitos urbanos

La violencia puede ser analizada desde múltiples ángulos y en sus diferentes dimensiones: violencia política o la familiar, violencia de masas o violencia individual. Este trabajo explora una relación particular: la que se establece entre la construcción de la ciudadanía social y las diversas manifestaciones de la violencia urbana, en las principales ciudades centroamericanas en las últimas décadas, con el objetivo de contribuir a la construcción de una nueva cultura urbana donde prime la tolerancia.

La cuestión de la ciudadanía social ha tendido a ocupar cada vez más un importante espacio en la investigación y el debate en distintos ámbitos, debido a la complejidad de los procesos de democratización política y la persistencia de los obstáculos que enfrenta el respeto a los derechos humanos en varios países centroamericanos en los años recientes.

* Este artículo está basado en un trabajo elaborado para el Instituto de Estudios Iberoamericanos de Hamburgo, Alemania. Los autores son investigadores y docentes de la $U C A$. 
Como han expresado varios autores apoyándose en las ideas de Marshall (1964), la ciudadanía en general es el principio de la participación plena en un Estado, en donde los derechos civiles y la igualdad constituyen elementos claves; la participación política efectiva es otra dimensión fundamental; así como los derechos y las responsabilidades sociales.

Estos últimos despiertan el interés de los individuos por sus comunidades y crean vínculos de solidaridad a escala local y nacional (y en estos momentos también a escala transnacional debido a la globalización). En este sentido, la ciudadanía social se diferencia de la ciudadanía civil y de la ciudadanía política en que los derechos y deberes asociados a la primera son menos individuales y mas colectivos, y constituye un medio necesario y poderoso para luchar por la integración y contra la exclusión social (Roberts, 1998). Este planteamiento permite vincular una problemática crucial de las ciudades centroamericanas en los años actuales, la violencia urbana, con el proceso de construcción de ciudadanía social y una cultura urbana de tolerancia.

Así como los vínculos entre lo individual y lo colectivo pasan por la construcción de un sentido de comunidad, pensamos que la relación entre construcción de ciudadanía social y violencia urbana está estrechamente ligada a la existencia de comunidades en las ciudades, lo que permitiría potenciar la primera y reducir la segunda.

Es necesario aclarar, sin embargo, que frecuentemente se habla de la existencia de comunidades, olvidando que no todo grupo social, no cualquier agrupamiento de individuos y familias, constituye una comunidad. Para que exista una comunidad es necesaria la presencia de valores y aspiraciones sociales compartidos de carácter permanente, lo que va más allá de compartir intereses alrededor de problemas de carácter coyuntural.

La vinculación entre individuo y comunidad esta mediada por la familia (en sentido amplio y no tradicional de la misma), por la estructura de clases sociales, y las instituciones del Estado y la sociedad civil. Esto condujo a plantear que esta vinculación contiene tres dimensiones: la relación entre individuo y sociedad; la relación entre individuo y grupo; y la relación entre individuo y masa (Heller, 1985).

En el caso de sociedades complejas la primera relación asume la forma de una estructura de comunidades orgánicas de intereses con- 
tradictorios, ya que sólo cuando el nivel de diferenciación social es elemental una comunidad se puede identificar plenamente con una sociedad. Estas comunidades orgánicas no corresponden necesariamente a las clases sociales fundamentales. Por otra parte, la relación entre individuo y grupo se basa en factores coyunturales, por lo que no todo grupo llega a constituirse en una comunidad a menos que los factores causales que conforman el grupo adquieran un carácter estructural, permanente y la asociación sea conscientemente asumida. Finalmente, la relación entre individuo y masa se caracteriza por el carácter no estructurado de esta última, aunque en algunos momentos las comunidades asumen un comportamiento de masas.

La comunidad, según Heller, es una unidad estructurada, orgánica, integrada por grupos sociales con una jerarquía homogénea de valores al cual pertenecen necesariamente los individuos al haber nacido o ser proyectados en ella, o al hacer una elección relativamente autónoma por parte de individuos ya desarrollados. No obstante, la pertenencia a una comunidad no es obligatoria, ya que el individuo nace en una familia no en una comunidad.

La construcción de comunidades implica, además, la existencia y reconfiguración permanente de identidades sociales, particularmente las identidades urbanas en la problemática que nos ocupa, cuestión de singular importancia. Se han distinguido tres formas y orígenes de la construcción de la identidad social en los contextos urbanos actuales (Castells, 1998):

- Identidad legitimadora: introducida por las instituciones dominantes de la sociedad para extender y racionalizar su dominación frente a los actores sociales.

- Identidad de resistencia: generada por aquellos actores que se encuentran en posiciones/condiciones devaluadas o estigmatizadas por la lógica de la dominación, por lo que construyen trincheras de resistencia y supervivencia basándose en principios diferentes $u$ opuestos a los que impregnan las instituciones de la sociedad.

- Identidad de proyecto: cuando los actores sociales, basándose en los materiales culturales que disponen, construyen una nueva identidad que redefine su posición en la sociedad y, al hacerlo, buscan la transformación de toda la estructura social. 
Adicionalmente, tanto la construcción de la ciudadanía social como la violencia urbana están fuertemente influenciadas por el reforzamiento de la esfera del dominio privado y el debilitamiento de la esfera de lo público, que tiende al predominio de la subjetividad sobre la ciudadanía, por lo que se requiere de una nueva concepción de la democracia que enfrente la visión liberal predominante y permita reconstruir el concepto de ciudadanía social que vincule al Estado con los ciudadanos y a éstos entre ellos mismos (de Souza, 1991). La ciudadanía social constituye así una construcción histórica construida a partir de un proceso de inclusión progresiva y de adquisición de poder por la sociedad.

Esto supone una reivindicación colectiva de los derechos $y$ deberes civiles. La construcción de ciudadanía social exige la existencia de luchas y movimientos sociales que demanden al Estado el mantenimiento y la posible ampliación de los derechos ciudadanos, es decir, de un amplio $y$ fuerte proceso de inclusión de diversas voces capaces de reivindicar la integración e inclusión social. También la construcción de la ciudadanía social conlleva, además de la inclusión, la división de lo social en dos dimensiones: lo público y lo privado.

Lo público es, en palabras de Habermas (1981), ese “espacio/núcleo productor de sentido social en donde se discuten los problemas sociales y políticos que atañen a un colectivo". Para este pensador la modernidad se presenta como la génesis de una esfera pública y autónoma de deliberación política constituida por sujetos capaces de argumentar racionalmente en condiciones de igualdad de participación y de réplica. Esta participación es la que constituye uno de los elementos que fortalecen las construcciones democráticas. En este espacio es donde se hacen las demandas sociales, es decir, donde se hacen visibles los distintos grupos y discursos que conforman lo social. Acá se generan el conjunto de mecanismos para tratar los problemas colectivos. Lo privado, por otra parte, es el lugar en el que se generan las relaciones específicamente individuales. Con esta definición no se quiere oponer lo público y lo privado como dos situaciones ajenas entre sí, sino más bien articuladas como elementos de un mismo fenómeno: la construcción de ciudadanía social.

El tránsito por diversas formas de violencia, en el que se han cuestionado los principios de ciudadanía y democracia, ha sido una de las características de la historia centroamericana. La discusión sobre la 
construcción de ciudadanía se vuelve, pues, una discusión pertinente en los momentos actuales, ya que a una década de la finalización formal de conflictos internos, todos los países del istmo presentan niveles bajos de participación social, un criterio importante en la construcción ciudadanía, y altos niveles de violencia, en sus diversas manifestaciones.

Este enfoque permite analizar las diversas manifestaciones de la violencia urbana en las ultimas décadas y la situación actual en algunas de las principales ciudades centroamericanas:

\begin{tabular}{|c|c|}
\hline $\begin{array}{l}\text { REQUISITOS PARA LA CONSTRUCCIÓN } \\
\text { DE CIUDADANÍA SOCIAL }\end{array}$ & $\begin{array}{l}\text { ASPECTOS A ANALIZAR RESPECTO A LAS } \\
\text { EXPRESIONES DE VIOLENCIA URBANA }\end{array}$ \\
\hline $\begin{array}{l}\text { Grado de presencia de } \\
\text { comunidades }\end{array}$ & $\begin{array}{l}\text { Relación con la presencia de } \\
\text { comunidades urbanas }\end{array}$ \\
\hline \multirow{2}{*}{$\begin{array}{l}\text { Mediación entre individuo y } \\
\text { comunidad } \\
\text { - Familia }\end{array}$} & \\
\hline & $\begin{array}{l}\text { Relación con las transformaciones } \\
\text { en las relaciones familiares }\end{array}$ \\
\hline —clases sociales & $\begin{array}{l}\text { Relación con la actual estructura } \\
\text { de clases sociales }\end{array}$ \\
\hline —instituciones estatales & Relación con las acciones estatales \\
\hline $\begin{array}{l}\text {-organizaciones de la } \\
\text { sociedad civil }\end{array}$ & $\begin{array}{l}\text { Relación con las actividades de la } \\
\text { sociedad civil }\end{array}$ \\
\hline $\begin{array}{l}\text { Existencia de identidades } \\
\text { sociales }\end{array}$ & $\begin{array}{l}\text { Relación con la existencia de } \\
\text { identidades sociales urbanas }\end{array}$ \\
\hline Relación público/privado & $\begin{array}{l}\text { Relación con el nivel de } \\
\text { privatización de servicios y } \\
\text { espacios públicos }\end{array}$ \\
\hline
\end{tabular}


2. La violencia en Centroamérica: una continuidad explicada por la historia

\section{Primer acto: la violencia previa a la década de los 80}

Antes de los 80, en Centroamérica prevalecía una constante violencia institucionalizada de carácter político promovida por gobiernos militares. Las otras manifestaciones de violencia se desdibujaban en un ambiente convulso estimulado por la desigual distribución de la riqueza y la preeminencia de la exclusión social. Estos factores internos se acentuaban por la sombra generada en todo el mundo por la guerra fría y el temor generalizado a la expansión del comunismo. Costa Rica constituía la excepción.

En este país se aplicó, desde 1950, una política social basada en un régimen político democrático y en la existencia de una distribución de la riqueza con pocas desigualdades comparada con las de sus vecinos de la región. El Estado de Bienestar costarricense dio lugar a una continuidad democrática que ha tenido su fundamento en relaciones solidarias y pacíficas entre los individuos. Sumado a esto, se han propiciado espacios y mecanismos de resolución de conflictos que explican los niveles bajos de inseguridad y violencia. Pese a su estabilidad interna, Costa Rica sería afectada desde los años 80 por las repercusiones de los conflictos internos vividos en los otros países de la región y una profunda crisis económica.

En Guatemala, el conflicto armado iniciado en la década de los 1960 se superpone a una ancestral violencia basada en la explotación de la población indígena, mayoritaria en el país. El profundo e irresuelto conflicto entre ésta y la población de origen ladino dio origen a altos niveles de violencia de todo tipo, entre la que se destacan la exclusión económica y social de los indígenas y la represión política (Jonas, 1994). Es este país en donde la violencia está profundamente teñida de un rasgo étnico de una importancia que no existe en el resto de países centroamericanos.

El Salvador se caracteriza desde el siglo XIX por una recurrente violencia contra la población campesina y la represión política contra todos los opositores a los gobiernos dictatoriales (Alvarenga, 1996). Esta situación, sumada a la violencia de orden criminal común se mantiene desde esa época. En la década del 70, las crecientes expresiones 
populares de protesta tuvieron como escenario todo el país, generando una feroz represión política que presagiaba la guerra que se desplegó la década siguiente.

En Nicaragua, la violencia cotidiana tiene como trasfondo, hasta los años 70, el irresuelto conflicto entre liberales y conservadores, y a partir de esa década, entre ambos sectores políticos y los sandinistas, que impulsaron varias insurrecciones urbanas hasta derrocar a la dictadura de la familia Somoza. (Cuadra, 2001). Hasta el momento actual, la persistencia del conflicto ha conducido a una situación de ingobernabilidad política que contribuye al nivel de violencia existente, en el que las motivaciones políticas se superponen a las contradicciones entre grupos familiares y económicos.

Honduras tampoco ha estado exenta de manifestaciones de violencia, muchas de ellas vinculadas a razones políticas. Ocurrió un masivo exilio de opositores durante la dictadura de 1933 a 1949 y durante el golpe de Estado de 1963. En la década de los setenta la represión pasó golpear los sectores sociales del campo. La forma en que se concretizó fueron las torturas y desapariciones de una cantidad no cuantificada de campesinos (Castellanos, 2000).

En síntesis, Centroamérica se caracteriza antes de los años 80 por la presencia de formas tradicionales de violencia subsumidas dentro de una permanente e intensa violencia de orden político. El tipo de regímenes políticos vigentes, donde el papel de las fuerzas armadas es dominante, explica esta situación. La diferencia de Costa Rica se explica además de una mayor equidad en la distribución de la riqueza, por el predominio de la democracia liberal como sistema político y a la abolición del ejército en 1950.

\section{Segundo acto: la década de las guerras}

A partir de los años 1980, Centroamérica será conocida internacionalmente como una región de guerras civiles, transformándose las manifestaciones de la violencia y emergiendo la cuestión de los derechos humanos a un primer plano.

En El Salvador, el conflicto armado abierto duró once años y se generalizó a todo el país con una pérdida en vidas de 75,000 personas aproximadamente. Más allá de su dimensión cuantitativa, la guerra se convirtió en un evento totalizante en el orden social del país (Martín 
Baró, 1988). La vida nacional estuvo en función del conflicto, en mayor o menor medida a lo largo de todo este período.

La ciudad de Guatemala fue el principal escenario de la lucha entre el gobierno y la oposición a inicios de la década de los ochenta. La dictadura militar lanzó un ataque de grandes proporciones en contra de los sindicalistas, estudiantes, intelectuales y todos aquellos que participaban en la oposición. La ciudad capital, lugar en el que se concentraba el poder y por muchos años la oposición al gobierno, se convirtió en un lugar inseguro para los activistas del movimiento popular (Ball et al, 1998). Una vez reducida la presencia de oposición política en la ciudad y replegada esta en el interior, la violencia política se trasladó a las zonas rurales.

Aunque en Honduras no se vivió un clima de guerra civil como Guatemala y El Salvador, sí se presentaron crecientes hechos de violencia. Debido a los conflictos en los países vecinos y al interés norteamericano en neutralizarlos, la presencia militar norteamericana tomó dimensiones considerables. Esto hizo que la sociedad hondureña se militarizara. Las expresiones de violencia más frecuentes estaban vinculadas a daños contra la vida, contra la propiedad y delitos varios, como amenazas, lesiones, violencia intrafamiliar, allanamientos, etc.

En Nicaragua, el gobierno sandinista no pudo evitar los ataques contrarrevolucionarios apoyados por los Estados Unidos. La guerra marcó toda la década y acentuó la extrema polarización política que se mantiene aún, la cual constituye una de las bases de la permanente inestabilidad política del país. Esta situación deterioró la economía y acentuó la pobreza, generando nuevas fuentes para la emergencia de diversas formas de violencia. La percepción de inseguridad difería según los espacios geográficos y la posición de los individuos respecto al conflicto.

En Costa Rica, en 1980, se da una ruptura de la estabilidad social provocada por una profunda crisis económica. Se experimenta un debilitamiento de las políticas sociales y crecimiento de las desigualdades y la pobreza provocando un crecimiento de la delincuencia y la violencia. Esta situación se agrava por la debilidad en los mecanismos de administración de justicia. Para mediados de la década de los ochenta, los datos comienzan a mostrar el crecimiento en el total de los delitos cometidos (Loría, 2001). 
En general, durante esta década la violencia alcanzó en Centroamérica niveles antes no vistos e inéditas manifestaciones. Los conflictos, como lo hemos señalado, eran, sobre todo, por razones políticas. Frecuentes denuncias de violación a los derechos fundamentales fueron hechas por organismos nacionales e internacionales.

\section{Tercer acto: ¿violencia en tiempo de paz?}

La década de los noventa se inicia con procesos de negociación en los países en los que el conflicto armado se había desarrollado. En El Salvador y Guatemala se lograron, a inicios y mediados de esta década, firmar acuerdos de paz y comenzar procesos de transición democrática. Esto no supuso, sin embargo, la disminución de la violencia. En todos los países centroamericanos se incrementaron los índices de lo que especialistas han denominado violencia social (Cruz y González, 2002). Esta se caracteriza por un aumento de la criminalidad expresada en delincuencia común y organizada.

Los procesos de criminalidad no están separados de la victimización a la que son expuestos los ciudadanos. La victimización es el acto en el cual una persona es objeto del uso de la fuerza provocada por la violencia que produce daño físico o psicológico (Cruz, M. 1999). Muchas veces, la sensación de inseguridad se percibe con mayores dimensiones por la cobertura que se da de estos hechos en los medios de comunicación social. Éstos han jugado un papel muy importante en la configuración de miedos urbanos, en los que la violencia o el sentimiento de inseguridad ocupan lugares prioritarios en la cobertura de noticias.

En Guatemala en 1996 la Unidad Revolucionaria Nacional Guatemalteca (URNG), y el gobierno firmaron un acuerdo de paz que permitió poner un final de la guerra. Los años que siguieron se han caracterizado por una presencia importante de la violencia como fenómeno social. Debido a esta situación, la inseguridad se ha convertido en uno de los principales problemas para la población guatemalteca. La demanda de la sociedad civil gira, consecuentemente, alrededor de esta problemática. La violencia social y urbana se dan, en Guatemala, principalmente por la amplia disponibilidad ilegal de armas, la persistente desigualdad social, la debilidad del sistema de administración de justicia y ante todo por cultura de la violencia que se basa en la anti- 
gua y arraigada exclusión social de la población indígena. (Rodríguez y De León, 2001).

En El Salvador, la situación es semejante. Las formas de violencia más frecuentes son los robos, la extorsión, las heridas con arma blanca o de fuego, las amenazas de muerte, los secuestros y los homicidios. Además de estas manifestaciones, vinculadas directamente con la delincuencia, el país ha sido escenario de muertes producidas por conflictos entre pandillas juveniles (Lungo y Baires, 1994). Desde 1995 se ha venido registrando un incremento en la demanda ciudadana por combatir la delincuencia. La campaña ha sido conducida por los empresarios, industriales, el gobierno central y los medios de comunicación. La petición se centra en aumentar las penas a los delincuentes considerando que la modernización del sistema de justicia impulsada a raíz de los Acuerdos de Paz es una muestra de debilidad institucional. La prensa tiene como objetivo de sus acusaciones a los jóvenes de las pandillas juveniles, pertenecientes a sectores pobres de la población localizados en las zonas marginales de la ciudad.

Nicaragua inició la década de los noventa con demandas internas y externas por terminar el conflicto armado. El proceso de negociación que permitiría iniciar un proceso de transición democrática coincidió con el cambio de gobierno. Este proceso se caracterizó por un débil sistema de administración de justicia, una ausencia de proyecto político y de una gobernabilidad democrática Todos estos elementos contribuyeron a que la institucionalidad existente fuera rebasada por la intensidad de los conflictos y las expresiones de violencia. Si bien no existía, entonces, un conflicto armado como el de la década anterior, la estabilidad política no se pudo concretar. Los mecanismos utilizados, en muchas ocasiones para resolver los conflictos que aparecían tomaron un cariz coercitivo y policial ante un fenómeno que, al igual que en los otros países centroamericanos, tiende a ser cada día más predominantemente urbano.

Costa Rica es un caso interesante respecto a la violencia. Este país presenta, a partir de diversos estudios, uno de los niveles más bajos de criminalidad en América Latina, sin embargo, la sensación de inseguridad es muy alta (Carvajal, 2003). Uno de los factores que ha propiciado esta percepción han sido los medios de comunicación social. Sin embargo, pese a una tradición democrática muy fuerte, Costa Rica 
sufrió un aumento considerable, según sus parámetros, en los niveles de criminalidad. Desde inicio de la década, las denuncias hechas al ministerio público han ido en aumento.

Situación paradójica, entonces, la de Centroamérica. A mayor democracia mayores niveles de violencia ¿Cuál es la explicación de este fenómeno?

Planteamos la hipótesis según la cual la explicación es múltiple, debido a que, a pesar de la generalizada democratización experimentada por los países centroamericanos sometidos antes a regímenes dictatoriales, las historias y las culturas propias han creado contextos que explican la persistencia de la violencia, y que a pesar de que sus manifestaciones actuales sean semejantes, estos contextos son particulares, en un ejemplo de que la globalización no puede borrar las especificidades nacionales a pesar trascienda las fronteras de los países.

\section{El telón de fondo de la violencia en ciudades centroamericanas}

Las ciudades son escenarios de múltiples y variadas relaciones donde los conflictos inherentes a la vida social pueden expresarse en forma abierta e incluso en actos de violencia cuando no se logran resolver pacíficamente (De Roux, 1994). La violencia ha sido definida, en su acepción más general como el uso o amenaza de uso de la fuerza física con la intención de afectar el patrimonio, lesionar o matar a otro o a uno mismo (Briceño, 1997). Este concepto parece pertinente al momento de reflexionar sobre el fenómeno de la violencia en contextos urbanos. Aunque la mayor parte de la violencia ejercida contra una persona tiene una dimensión delictiva, y por lo tanto está penada socialmente, usualmente se ha adoptado el término de violencia urbana para hacer referencia al crimen cometido en los espacios públicos de las ciudades.

A pesar de la unidad geográfica los países de la región centroamericana y de su historia colonial común, éstos presentan particularidades sociales, políticas y culturales que se evidencian al observar las manifestaciones de violencia urbana en sus principales ciudades en el momento actual.

Analizar la violencia urbana en las ciudades centroamericanas exige tomar en cuenta el creciente proceso de urbanización que todos los 
países de istmo han vivido desde la industrialización de sus economías en la década de los 50. Así, en Nicaragua entre 1940 y 1995 el porcentaje de población que vivía en las ciudades se elevó de $30 \%$ a $57 \%$. En El Salvador en 1996, 56,7\% de la población vivía en áreas urbanas y $43,3 \%$ en áreas rurales, aunque estos porcentajes ocultan el hecho de que buena parte de los habitantes censados como rurales trabajan en las ciudades. En Costa Rica, en 1995, el 49\% de los habitantes vivían en zonas urbanas. En Honduras, la población urbana representaba, en 1995, el $43 \%$ de la población total y se concentraba en dos ciudades: Tegucigalpa y San Pedro Sula. De los países del istmo, el que presenta una menor proporción de la población viviendo en ciudades es Guatemala. En 1995 este país solo contaba con un total de 35\% de población urbana.

Destaquemos los procesos explicativos emblemáticos para cuatro de las ciudades capitales que permiten recoger la diversidad de las manifestaciones de violencia urbana en la región centroamericana. Así, en el caso de San Salvador hemos escogido la temática de las expectativas surgidas a raíz de la firma de los Acuerdos de Paz de 1992 que dieron fin al conflicto armado de más de diez años; en la ciudad de Guatemala la profunda exclusión social de la población indígena; en Managua la persistente inestabilidad política; y en San José los efectos de la intensa migración nicaraguense.

San Salvador, la frustración luego de los Acuerdos de Paz

La guerra que asoló a El Salvador entre 1981 y 1992 dejó, evidentemente, secuelas en el comportamiento social que constituyen fuente de violencia. Se destacan la desconfianza y los rencores entre los distintos grupos sociales involucrados en el conflicto a pesar de haberse logrado firmar un ejemplar acuerdo de paz (Samayoa, 2002); el rompimiento de la estructura familiar tradicional; la existencia de una gran cantidad de armas entre la población civil y la capacidad adquirida para utilizarlas; entre otras.

Por estas razones surgió inmediatamente el temor a un incremento de la violencia, lo que efectivamente ocurrió y fue atribuido rápidamente a los factores antes mencionados, especialmente al último. No obstante, al analizar la violencia desarrollada en los últimos diez años es indispensable buscar las razones estructurales que la originan. Sostenemos que una de las fuentes fundamentales en el caso salvadoreño es 
la frustración surgida al constatar que los Acuerdos de Paz sólo han cambiado, parcialmente, las condiciones que provocaron el conflicto armado.

Efectivamente, aunque es innegable que el país se ha democratizado en el aspecto político, la concentración de la economía y la desigualdad social se han acentuado durante la última década. El nuevo modelo económico descansa en cuatro bases: la existencia de un importante excedente externo constituido por las remesas de los migrantes; la estabilidad cambiaria y la reducción de la inflación a niveles internacionalmente aceptados; una nueva forma de integración de la economía salvadoreña a la norteamericana; y la expansión del mercado interno. Aunque aún está en proceso de consolidación, este nuevo modelo económico ha generado ya ganadores y perdedores. Entre los primeros está el sector financiero, las empresas transnacionales y los industriales vinculados a la maquila. Entre los segundos se encuentran los trabajadores del campo y los agroexportadores (Segovia, 2002).

A pesar que entre 1996 y el 2000 disminuyó la pobreza y se incrementó la cobertura de los servicios básicos, el crecimiento económico se detuvo, el subempleo se mantuvo alto y los salarios mínimos se redujeron. El Salvador presenta una de las desigualdades más altas del mundo en la distribución del ingreso. El 20\% más rico de la población percibe en promedio ingresos 18 veces más altos que el $20 \%$ más pobre. Existe por ello un ambiente de incertidumbre e inseguridad hacia el futuro (PNUD, 2001). Una investigación reciente indica como durante la última década el poder económico ha permanecido concentrado como en las décadas precedentes a la guerra de los años 80 (Paniagua, 2002).

Esta situación ha incrementado la migración de los salvadoreños hacia el exterior, con todas las consecuencias positivas y negativas implícitas para la transformación de la sociedad nacional, destacándose entre las últimas el fenómeno de las pandillas juveniles influenciadas por el proceso migratorio, conocidas como "maras". No obstante, la relación entre los impactos de la intensa migración internacional de los salvadoreños (el $25 \%$ de la población vive actualmente en el exterior), no debe hacer ver a la creciente violencia como un efecto unidirecccional de este proceso. Investigaciones realizadas muestran la gran complejidad de este fenómeno en sus dimensiones sociales y culturales (Lungo y Kandel, 1997). 
Una investigación sobre este fenómeno mostró que el 75\% de los miembros de estas pandillas se encuentran desempleados, casi la mitad buscó empleo en las semanas previas a la encuesta y a lo que aspiran es a obtener uno; que el $10 \%$ participó en la guerra en alguno de los bandos contendientes; y que tienen pocas expectativas en el futuro del país (Cruz y Portillo, 1998), lo que indica que, a pesar de las actitudes ambivalentes de los miembros de las pandillas, la incertidumbre sobre el futuro estimula la violencia urbana de éste y otros sectores sociales.

La generalizada frustración por los resultados de los Acuerdos de $\mathrm{Paz}$ en el caso salvadoreño es uno de los procesos fundamentales que incrementa la violencia urbana en sus distintas manifestaciones, especialmente en el Área Metropolitana de San Salvador. Las encuestas en torno a las elecciones para los gobiernos locales a realizarse en marzo del 2003 muestran que este problema, junto al deterioro de las condiciones económicas experimentadas por los sectores populares y las clases medias urbanas, constituyen las principales preocupaciones de los ciudadanos.

\section{Guatemala, el peso de la exclusión social de la población indígena}

La sociedad guatemalteca constituye la expresión más impactante en la región centroamericana del fenómeno de la exclusión de la población indígena en sus diferentes dimensiones (Bastos y Camus, 1994). Desde los primeros estudios sobre la migración hacía la ciudad de Guatemala a finales de los años 60 (Roberts, 1968), pasando por el exhaustivo análisis de décadas de violencia política (Jonas, 1994), hasta investigaciones recientes sobre sectores sociales específicos (Geller y Palma, 1999), todos los trabajos han documentado la profundidad de este fenómeno, una de las fuentes fundamentales de la violencia urbana en la ciudad de Guatemala.

El proceso emblemático que contextualiza la violencia urbana en la ciudad de Guatemala ( $y$ en general en todo el pais), es la exclusión social de la población indígena. Esta vive en las peores condiciones de pobre$\mathrm{za}$, a lo que se suma, en el ámbito urbano el desarraigo y las enormes dificultades para adaptar a la vida de la ciudad. Las razones se encuentran en el peso de su tradición campesina, ser mayoritariamente analfabetos y mayahablantes (de diferentes lenguas, adicionalmente), y el hecho fundamental de ser desplazados por la violencia política y el miedo de sus zonas de origen, llegando a la ciudad por obligación y 
sin ninguna expectativa previamente construida socialmente. Por esta razón privilegian en ocultamiento, diluyéndose en la ciudad, sin mantener ni recrear el rico mundo comunitario vivido anteriormente en el campo (Smith, 1992; Bastos y Camus, 1994).

Cuestiones como el inquilinato son, para esta nueva población de la ciudad, totalmente incomprensibles. Respecto a la inserción laboral, obviamente las actividades de la economía informal conforman casi la única opción, acentuando así la exclusión social y siendo objeto privilegiado de la violencia urbana.

La firma del Acuerdo de Paz en Guatemala no ha contribuido, lamentablemente, a la transformación de esta situación. Al contrario, las dificultades de construir una gobernabilidad democrática durante los últimos seis años se ha sumado a la expansión del crimen organizado, incrementando la violencia urbana en la ciudad y afectando a la población más vulnerable, los pobres, en su mayoría indígenas, particularmente a las mujeres de este origen étnico.

Este panorama se revela como un desafío, en el caso de la ciudad de Guatemala, la reducción socialmente sostenible de la violencia urbana sin hacer que previamente la población indígena adquiera plenamente los derechos que implica una real ciudadanía social.

Managua, un escenario permanente de inestabilidad politica

Un rasgo esencial que particulariza a la sociedad nicaraguense desde el siglo XIX es la permanente inestabilidad política. Basada en el antiguo e irresuelto conflicto entre liberales y conservadores, pasa por la ocupación e intervención militar norteamericana y se acentúa por la revolución Sandinista. El desenlace aún esperado de esta permanente inestabilidad política al iniciarse el siglo XXI, marca la atmósfera de violencia urbana en el país y en Managua.

La selección de esta ciudad como capital del país obedece, entre otras razones de mayor importancia como el desarrollo de la economía, a la rivalidad entre las dos ciudades durante la colonia: León, la liberal, y Granada, la conservadora, que ha sido sujeto de varias obras literarias. Subyace en este hecho de la geografía urbana del país, una profunda contradicción entre intereses económicos que adquirió la expresión de rivalidades familiares que aún cruzan la política nicaragüense y que no pudieron ser borrados ni aún durante la década del 
gobierno sandinista. Managua, de tardía consolidación como la principal ciudad del país, será rápidamente el escenario privilegiado de la violencia urbana.

Esta asume, especialmente durante la década de los 70 un tinte eminentemente político. La ciudad es el lugar de varias insurrecciones populares contra la dinastía de la familia Somoza (Barreto, 80), dejando una profunda huella en numerosos barrios de la ciudad.

Aunque durante los años del gobierno sandinista la violencia política se traslada hacia las zonas rurales y en general la violencia común disminuye sensiblemente, al ser derrotados los sandinistas en las elecciones de 1990, vuelve a emerger con fuerza la permanente inestabilidad política que caracteriza a la bistoria nicaraguense. Derrotada la ilusión revolucionaria se vuelve a la cotidiana negociación poco transparente entre los partidos políticos en momentos en que la acentuada pobreza heredada por diez años de guerra interna y la crisis económica conducen al surgimiento de varias formas de violencia urbana entre las que se destaca el crimen organizado. El país tampoco es ajeno a las consecuencias de la migración internacional. Así, las ciudades de Managua, Masaya y Chinandega son las que más concentran pandillas juveniles (Valle y Argüello, 2002).

Pareciera, entonces que una condición indispensable para la construcción de ciudadanía social y la reducción sostenida de la violencia urbana pasan por la solución de la permanente inestabilidad política del país.

San José, el impacto de la migración nicaraguense

A pesar de su conocida democracia política, su relativa igualdad social, un nivel de pobreza relativamente bajo y un ambiente pacífico donde las manifestaciones de violencia que han caracterizado a las otras ciudades centroamericanas no han estado presentes, el panorama en la ciudad de San José ha cambiado drásticamente en los años recientes.

Si en los años 80 la crisis económica que erosionó profundamente las bases de la sociedad costarricense tuvieron su expresión en el incremento de la informalidad de la economía de la ciudad, el surgimiento de un importante sector informal de la economía y la aparición del fenómeno de la segregación socio-espacial (Lungo, 1997), 
constituyéndose en la base de manifestaciones de violencia urbana antes no vistas, será un nuevo fenómeno en los años 90 el que, sumado a lo anterior, explica que la atmósfera pacífica de la ciudad esté rápidamente desapareciendo.

Este fenómeno, estrechamente asociado a la situación que vive el vecino país de Nicaragua, y que hemos descrito refiriéndonos a la ciudad de Managua, es el intenso flujo migratorio de nicaragüenses que se asientan, ante todo, en el valle central y especialmente en la ciudad de San José. Este proceso no es nuevo. Varios estudios han detectado la concentración de migrantes nicaraguenses en algunos barrios populares de San José como Barrio Cuba, y su dedicación especialmente a trabajos artesanales especializados como la fabricación artesanal de calzado. Sin embargo durante los años 90 la migración de nicaraguenses al país aumentó drásticamente, estimándose que su número alcanza cerca de medio millón de personas, cifra alta en relación a un país que solo cuenta con cuatro millones de habitantes.

Alrededor de la explicación de sus causas hay un intenso debate que lamentablemente atribuye muchas de las manifestaciones de la creciente violencia urbana a los migrantes nicaraguenses. Se ha llegado a sostener que incluso estas se generan en algunos asentamientos urbanos precarios habitados mayoritariamente por población originaria de este país.

Este caso ejemplifica la relación, frecuentemente mal entendida, entre la migración extranjera y la violencia urbana. Estados Unidos constituye una muestra paradigmática de esta distorsión de las razones que provocan las nuevas y a veces sofisticadas manifestaciones de la misma.

\section{El desafío: reducir la violencia urbana construyendo ciudadanía social en el contexto de la globalización actual}

En contextos en el que el sentimiento de inseguridad, desconfianza, temor por la ciudad misma que se presenta llena de amenazas, ¿cómo construir ciudadanía social? Para responder a esta interrogante, se retomará el planteamiento hecho en la introducción: la ciudadanía supone y exige la presencia de cuatro elementos constituyentes: la existencia de comunidades urbanas; el manejo de las distintas mediaciones que afectan la relación entre el individuo y estas comunidades;

\section{1}

Ciudadanía social y violencia en las ciudades centroamericanas 
la construcción de identidades sociales urbanas; y la relación entre lo público y lo privado. Lo anterior, contextualizado por el proceso de globalización actual.

\section{Existencia de comunidades urbanas}

En Centroamérica, el grado de presencia de comunidades urbanas fue un elemento importante en la década de los 80 . En este período las demandas sociales se hacían desde grupos organizados que, si bien no en todos los casos actuaban dentro del marco legal prevaleciente, permitín canalizar las luchas y aspiraciones por el reconocimiento de los derechos humanos.

En la década de los 90 , la construcción de comunidades urbanas ha sufrido transformaciones. Las ciudades centroamericanas, afectadas por elevados niveles de violencia urbana han propiciado el repliegue de los ciudadanos de todas las clases sociales a espacios que consideran seguros. Estos espacios son, predominantemente, espacios privados. La desconfianza en "el otro", la amenaza que éste representa, han venido a deteriorar las relaciones en las comunidades tradicionales. Esto es más evidente en las relaciones de comunidades de barrio. La proximidad del vecino, los espacios de socialización con éste se cierran a partir de la desconfianza que prima en las relaciones interpersonales, imponiendo patrones de conducta que obstaculizan la integración comunitaria y reducen los niveles de tolerancia a lo diferente.

En las distintas ciudades centroamericanas puede observarse cómo se han establecido relaciones más de carácter coyuntural que permanente en la búsqueda de mecanismos de seguridad común. Estas respuestas, más que fortalecer el concepto de comunidad como un espacio permanente en el que se comparten valores y aspiraciones sociales, se vuelven respuesta a mecanismos ineficientes de garantía de la seguridad por parte del Estado.

En este aspecto, puede afirmarse que existe en las ciudades centroamericanas un debilitamiento en la construcción de comunidades que contribuyan a la construcción de ciudadanía social y la reducción socialmente sostenible de la violencia urbana. Este proceso no aparece directamente vinculado a la globalización. 
La relación de los individuos con la comunidad opera a través de distintas mediaciones que afectan la construcción de la ciudadanía social y la lucha contra la violencia urbana: la familia, la estructura de clases sociales, las instituciones del Estado y la sociedad civil, expresada en organizaciones sociales

En Centroamérica, la estructura de las relaciones familiares ha experimentado transformaciones importantes en las últimas décadas. Algunos de los factores que han contribuido a esta transformación son la exclusión social, la pobreza y la migración internacional. Así por ejemplo, los rasgos principales de la familia nicaragüense pobre actual expresan el cambio del modelo familiar tradicional que tiene al hombre como centro. La jefatura femenina de las familias aumentó sobre todo en la ciudad de Managua (Valle y Argüello, 2002). Costa Rica registra en los últimos años una consolidación de la tendencia al aumento de los hogares unipersonales, nucleares conyugales sin hijos y nucleares uniparentales. Ambos casos muestran transformaciones en la estructura de las relaciones familiares que, indudablemente guardan relación con el fenómeno de la violencia urbana.

Una vez iniciada la década de los noventa, con las negociaciones que dieron fin a los conflictos, la polarización de la lucha de clases que había predominado en los años anteriores se desdibujó. La clase media surge como un referente y un elemento importante en la estructura social de las ciudades centroamericanas. Esta clase media ha sido determinante en la construcción de las sociedades urbanas que se han configurado en la región. En varios países fue de ellas donde surgieron los grupos de oposición al régimen militar de los años 70 y 80 . Este comportamiento, de mucha presencia y compromiso con lo público y político, en la actualidad tiende hacia un repliegue hacia los intereses privados principalmente.

Las acciones que desde ella se articulan están, ante todo, vinculados con la seguridad o el acceso a servicios urbanos, dado el hecho que los sectores medios se han quedado sin proyectos ideológicos y políticos en los cuales confiar y a través de los cuales encauzar demandas más integrales; por otra parte, la clase media ejemplifica, mejor que otro grupo social, por sus aspiraciones, ingresos, educación, la dinámica privatizadora que está más allá del miedo a la represión y al

\section{3}

\section{Ciudadanía social y violencia en las ciudades centroamericanas}


desorden (González, 2002), y que involuntariamente contribuye a la violencia urbana.

En general, las instituciones estatales se encuentran en un momento de deslegitimación social en Centroamérica. Con relación a la seguridad, pese a que se han impulsado programas de modernización de los sistemas de administración de justicia, éstos tienen aún una institucionalidad muy débil y sus mecanismos no son eficaces para solucionar los problemas de seguridad urbanos. Esto se pone en evidencia cuando este tema aparece como un problema a solucionar delegándolo en manos privadas.

$\mathrm{El}$ aumento de la presencia de servicios de seguridad privada es un hecho real y en proporciones alarmantes en la región. Así, en Costa Rica, el porcentaje de personal de seguridad privada inscrito se elevó entre 1994 a 1998 en un $268.58 \%$ (Loría, 2001). En Guatemala, para 1999, se reportaban 23 mil agentes de seguridad privados y la compra venta de servicios de seguridad, vigilancia y armas de fuego, se había incrementado en 50\% (Rodríguez y De León, 2001).

Respecto al gobierno de las ciudades prevalecen todavía niveles muy altos de centralización del Estado. Los procesos de descentralización o están en sus primeras etapas, como es el caso de Nicaragua, o están estancados, como en El Salvador, lo que pone en situación de desventaja el gobierno de las ciudades para contribuir a la construcción de ciudadanía y combatir la violencia urbana, que sigue estando, esta última tarea, en manos del gobierno central y en el que prevalece un enfoque policial reactivo.

Las organizaciones de la sociedad civil han tenido una participación muy activa en el fortalecimiento de comunidades en todos los países centroamericanos en los últimos años. Muchas de ellas trabajan por la reducción de la proliferación de armas, por la integración de jóvenes de pandillas a la vida comunitaria, por la apertura de espacios de esparcimiento, etc. No obstante, su excesiva fragmentación, el énfasis de corto plazo de sus proyectos y su dependencia de la fluctuación de las políticas de los donantes, reducen los efectos de su trabajo. Cuando se observa su papel en la formación y consolidación de comunidades y la articulación de éstas con las instituciones estatales, son visibles las limitaciones de su trabajo en torno a la violencia urbana. 
La identidad social se construye en interacción con otros sujetos que participan de características comunes, en función de su posicionamiento social: "nosotras las mujeres, nosotros los obreros, nosotros los jóvenes”. Las identidades sociales urbanas, son recortes construidos que responden a los modos de relación con la ciudad. Ésta se construye en la interacción cotidiana. Hablar de identidades es hablar de clase social, de grupos, de oficios, de nombres, de espacios y territorios.

Se pueden entender las identidades sociales desde una triple referencia, a saber, la situacional, la grupal y la simbólica (Reguillo, 1991). La referencia situacional lleva en sí misma la idea de espacios, escenarios, lugares sociales que introyectan en el actor social una idea de quién es, quién ha sido y cuáles son sus posibilidades reales. Esta referencia reconoce que "el lugar" (el espacio, el territorio), es determinante en los itinerarios cotidianos y es desde estos que se establecen relaciones con la ciudad. La referencia principal son los grupos a los que los actores sociales se adscriben y desde los que se da sentido al juego de posiciones sociales. La referencia simbólica retoma la manera en que se exterioriza la identidad social: ropa, accesorios, lenguaje, gustos, etc.

En las ciudades centroamericanas se han configurado, evidentemente, diversas identidades sociales urbanas que se reconfiguran constantemente. La transformación de las identidades es provocada por una multiplicidad de factores, entre los que podrían citarse las condiciones sociales y económicas de los distintos grupos, el proceso de la migración, la transformación de la ciudad, la presencia de los medios de comunicación en la vida cotidiana, etc. Una de las nuevas identidades sociales que pueden distinguirse en las ciudades centroamericanas es la conformada por las pandillas juveniles, que tiene una fuerte presencia en todas ellas.

Estamos, sin embargo, ante una de las cuestiones menos exploradas en evolución reciente de las ciudades de la región. Por ejemplo, habría que estudiar las transformaciones que sufre la identidad social de los grupos indígenas luego de varios años de vivir en la ciudad de Guatemala o la recreación de la identidad de los nicaragüenses que habitan en San José. 
La relación entre lo público y lo privado está vinculada con el concepto de democracia y por consiguiente con las cuestiones de la ciudadanía social y seguridad como sostuvimos al inicio. Lo público es el espacio/núcleo productor de sentido social en donde se discuten los problemas sociales y políticos que atañen a un colectivo (Habermas, 1981). La modernidad se presenta como la génesis de una esfera pública y autónoma de deliberación política constituida por sujetos capaces de argumentar racionalmente en condiciones de igualdad de participación y de réplica. Esta participación es la que constituye uno de los elementos que fortalecen las construcciones democráticas. En otro extremo de la construcción de ciudadanía, está el ámbito de lo privado. En este se expresan las demandas individuales de los sujetos.

La vida social pareciera moverse de uno a otro extremo. Pareciera que nuestras sociedades están predispuestas de algún modo hacia las oscilaciones entre períodos de intensa preocupación con los problemas públicos y de casi total concentración en las metas del mejoramiento individual y el bienestar privado (Hirschman, 1982). Este movimiento, que es una tendencia mundial reforzada por los procesos de globalización económica y cultural, parece ser el escenario de la relación con lo público en las ciudades centroamericanas.

La participación en asuntos de comunidad se restringen a la defensa de los privado. El objetivo es, al final, defender el territorio. Las soluciones a este sentimiento de inseguridad y desconfianza son las privatizaciones. Privatizaciones de espacios, de gustos, de relaciones. La violencia debilita lo público como instancia de socialización y se privilegia lo privado (Carrión, 1994)

Obviamente, esta tendencia tiende a que los ciudadanos no enfrenten colectivamente el problema de la violencia urbana, y cuando lo hacen enfatizan en opciones de seguridad privada, reduciendo el papel de lo público. Buena parte de los habitantes de las ciudades centroamericanas parecen regirse por este patrón de comportamiento.

En síntesis, podemos plantear que el proceso de globalización actual está introduciendo condicionantes comunes en las mediaciones entre los individuos y las comunidades, en la transformación de las identidades urbanas y en la relación entre lo público y lo privado en las sociedades urbanas centroamericanas. Sin embargo, la fuerza histó- 
rica de los contextos específicos mostrados anteriormente para El Salvador, Guatemala, Nicaragua y Costa Rica, muestran lo equivocado de las posiciones que sostienen que estamos ante un universo homogéneo, lo que hace que debamos incorporar en el análisis de la relación entre ciudadanía social y violencia urbana tanto los factores nacionales y locales, como los factores que genera la creciente transnacionalización de las sociedades centroamericanas, y las contradicciones entre ambos.

Para finalizar, proponemos un conjunto de criterios que podrían impulsarse para reducir la violencia tomando en consideración los principales problemas identificados las ciudades centroamericanas que tienen relación con la violencia urbana:

\begin{tabular}{|c|c|}
\hline $\begin{array}{l}\text { TENDENCIAS CLAVES EN CIUDADES } \\
\text { CENTROAMERICANAS }\end{array}$ & $\begin{array}{l}\text { CRITERIOS A CONSIDERAR EN EL } \\
\text { DISEÑO DE PROGRAMAS CONTRA LA } \\
\text { VIOLENCIA URBANA }\end{array}$ \\
\hline $\begin{array}{l}\text { - Permanencia de altos niveles } \\
\text { de pobreza }\end{array}$ & $\begin{array}{l}\text { - Combinar acciones que } \\
\text { generen ingresos con los } \\
\text { con los programas que buscan } \\
\text { reducir la violencia }\end{array}$ \\
\hline $\begin{array}{l}\text { - Acentuación de la segregación } \\
\text { socio-espacial }\end{array}$ & $\begin{array}{l}\text { - Crear y recuperar de nuevos } \\
\text { espacios públicos como eje } \\
\text { de ciertos programas } \\
\text { desfavorecidos }\end{array}$ \\
\hline $\begin{array}{l}\text { - Exclusión social de grupos } \\
\text { desfavorecidos (indígenas, } \\
\text { mujeres, jóvenes) }\end{array}$ & $\begin{array}{c}\text { - Incorporar acciones especiales } \\
\text { para reducir la violencia contra } \\
\text { los grupos más desfavorecidos }\end{array}$ \\
\hline $\begin{array}{l}\text { - Ausencia de formas de } \\
\text { gobernabilidad urbana } \\
\text { democráticas }\end{array}$ & $\begin{array}{l}\text { - Institucionalizar instancias } \\
\text { participativas ciudadanas en los } \\
\text { gobiernos locales para } \\
\text { implementar programas contra } \\
\text { la violencia }\end{array}$ \\
\hline $\begin{array}{l}\text { - Estigmatización y marginación } \\
\text { de los migrantes extranjeros y } \\
\text { los migrantes nacionales } \\
\text { retornados }\end{array}$ & $\begin{array}{l}\text { - Diseñar proyectos especiales } \\
\text { para incorporar a los migrantes } \\
\text { en los programas contra la } \\
\text { violencia }\end{array}$ \\
\hline
\end{tabular}




\section{Referencias}

Alvarenga, Patricia (1996): Cultura y ética de la violencia. El Salvador 1880-1932, EDUCA, San José.

Ball, P., Kobrak, P. y Spirer, H.F. (1998): Violencia Institucional en Guatemala 1960-1996, Centro Internacional para investigaciones en Derechos Humanos, Guatemala.

Barreto, Pablo (1980): El repliegue de Managua a Masaya, editorial Cartago, México.

Bastos, Santiago y Camus, Manuela (1994): Sombras de una batalla. Los desplazados por la violencia en la ciudad de Guatemala, FLACSO, Guatemala.

Briceño León, Roberto (1997): "La cultura emergente de la violencia en Caracas", Revista Venezolana de Economia y Ciencias Sociales Vol. 3, \# 2-3, Caracas.

Carrión, Fernando (1994): "De la violencia urbana a la convivencia ciudadana", en Concha Eastman, A. y Cobo, G. (editores), Ciudad $y$ violencias en América Latina, PGU, Quito.

Carvajal, Guillermo (2003): La violencia urbana en Costa Rica, editorial Cátedra, San José.

Castellanos, J. (2001): "Honduras: armamentismo y violencia", en El arsenal invisible. Armas livianas y seguridad ciudadana en la postguerra centroamericana, Fundación Arias para la Paz y el Progreso, San José, Costa Rica.

Castells, Manuel (1998): La Era de la Información. Economía, sociedad y cultura. Tomo II, Alianza Editores, Madrid.

Cruz, Miguel (1999): La victimización por violencia urbana: niveles y factores asociados en ciudades seleccionadas de América Latina y España, Serie Documentos Técnicos N\# 4, Proyecto Activa de la Coordinación de Investigaciones División de Salud y Desarrollo Humano de la OPS. Ver en http://www.paho.org/Spanish/HDP/ HDR/serie04composite.PDF

Cruz, Miguel y Portillo, Nelson (1998): Solidaridad y violencia en las pandillas del gran San Salvador, UCA editores, San Salvador.

Cruz, Miguel y González, Luis (2002): "La magnitud de la violencia", en L. González y R. Cardenal, El Salvador en transición, UCA editores, San Salvador. 
Cuadra Lira, E. (2001): "Proliferación y control de armas en Nicaragua”, El arsenal invisible. Armas livianas y seguridad ciudadana en la postguerra centroamericana, Fundación Arias para la Paz y el Progreso, San José, Costa Rica.

De Roux, G. (1994): "Ciudad y violencia en América Latina”, Cuadernos de salud y desarrollo Vol. 2, Bogotá.

Geller. Gisela y Palma, Irene (1999): Precariedad urbana, desarrollo comunitario y mujeres en el Area Metropolitana de Guatemala, FLACSO, Guatemala.

González, Luis (2002): “Clase media y cambio social”, en ECA \# 639640, San Salvador.

Habermas, J. (1981): Historia y crítica de la opinión pública, Gustavo Gilli, Barcelona.

Heller, Agnes (1985): Sociología y vida cotidiana, Ediciones Península, Barcelona.

Hirschman, Albert (1982): Interés privado y acción pública, Fondo de Cultura Económica, México D.F.

Jonas, Susanne (1994): La batalla por Guatemala, FLACSO / Nueva Sociedad, Guatemala.

Loría Ramírez, M.A. (2001): “Costa Rica: Diagnóstico de armas de fuego", en El arsenal invisible. Armas livianas y seguridad ciudadana en la postguerra centroamericana Fundación Arias para la $\mathrm{Paz}$ y el Progreso, San José, Costa Rica.

Lungo, Mario (1997): "Costa Rica: Dilemmas of the Urbanization in the $80^{\prime}$ 's", en Urban Caribbean, A.Portes et al (editors), The Johns Hopkins University Press, Baltimore.

Lungo, Mario y Kandel, Susan (1997): Transformando El Salvador. Migración, sociedad y cultura, FUNDE, San Salvador.

Lungo, Mario y Baires, Sonia (1994): "La delincuencia en San Salvador después de la guerra: ¿cuáles causas?, ¿cuáles planes para su control?", en Ciudad y violencias en América Latina, A. Eastman, F. Carrión y G. Cobo (editores), PGU, Quito.

Marshall, Thomas H. (1964): "Citizenship and Social Class", en Class, Citizenship and Social Development, T.H. Marshall (editor), The University of Chicago Press, chicago.

Martín Baró, Ignacio (1988): "La violencia política y la guerra como causas del trauma psicosocial de El Salvador", Revista de Psicología de El Salvador \# 7, San Salvador. 
Paniagua, Carlos (2002): "El bloque empresarial hegemónico salvadoreño", ECA \# 645, San Salvador.

PNUD (2001): Informe sobre Desarrollo Humano. El Salvador 2001, San Salvador.

Reguillo, R. (1991): En la calle otra vez. Las bandas juveniles. Identidad urbana y usos de la comunicación, ITESO, Guadalajara.

Roberts, Bryan (1968): "Politics in a Neighbourhood of Guatemala City", en Sociology 2.

Roberts, Bryan (1997): "Ciudadanía y política social en latinoamérica", en Ciudadanía y política social, B: Roberts (editor), FLACSO/ SSRC, San José.

Rodríguez, M. y De León Wantland, M. (2001): “Armas ligeras y violencia en Guatemala”, en El arsenal invisible. Armas livianas y seguridad ciudadana en la postguerra centroamericana, Fundación Arias para la Paz y el Progreso, San José, Costa Rica.

Samayoa, Salvador (2002): La reforma pactada, UCA editores, San Salvador.

Segovia, Alexander (2002): Transformación estructural y reforma económica en El Salvador, F y G editores, Guatemala.

Smith, Carol (1992): "Changing indian identity: Guatemala's violent transition to modernity”, en Guatemala indians and State, C. Smith (editor), University of Texas Press.

De Souza Santos, Boaventura (1991): "Subjectividade, cidadania e emancipação", en Critica de Ciencias Sociais \# 37.

Valle Martínez, M. y Argüello, A. (2002): Diagnóstico de seguridad ciudadana en Nicaragua. Documento de trabajo del proyecto "Apoyo a la implementacion de una estrategia de seguridad ciudadana en Nicaragua", PNUD, Managua.

Nota

1. El Social Science Research Council de New York, en asociación con FLACSO. Costa Rica, apoyó una serie de trabajos y seminarios durante 1997, que analizaron la cuestión de la ciudadanía social en relación a la política social, la descentralización, los mercados laborales, la migración internacional y la problemática indígena (ver Roberts, 1998). 